\title{
The positional and temporal running demands of elite inter-county camogie match play across 5-min intervals
}

\author{
Philip Connors $^{1}\left[\right.$ Des Earls ${ }^{1} \cdot$ Declan Browne $^{1} \cdot$ Paula Fitzpatrick $^{1} \cdot$ Paula Rankin $^{1}$
}

Received: 15 September 2021 / Accepted: 17 January 2022 / Published online: 11 February 2022

(c) The Author(s), under exclusive licence to Springer-Verlag Italia S.r.l., part of Springer Nature 2022

\begin{abstract}
Purpose Camogie is a native Irish female field sport game. This investigation aimed to establish elite camogie players' positional and temporal running demands across 5-min intervals during competitive match play.

Methods Thirty-one $(n=31)$ inter-county camogie players (age: $24 \pm 4$ years; height: $167.2 \pm 6.3 \mathrm{~cm}$; body mass: $67.0 \pm 7.5 \mathrm{~kg}$ ) from two squads wore commercially available 10-Hz Playertek GPS units (Playertek by Catapult, Australia) during Senior All-Ireland Championship games $(n=8)$. Players were categorised according to match positions (defenders, mid-fielders, forwards). Games were split into quarters, and subsequently into 5-min intervals for analysis (5-min intervals are more closely associated with small-sided games and training than half-by-half comparisons).

Results Analysis revealed significant positional and temporal differences to exist $(p<0.05)$. Mid-fielders covered greater total $(r=0.16-0.19)$ and relative distance $(r=0.15-0.17)$, with forwards covering greater high-speed distances $(r=0.09-0.19)$. Mid-fielders had greater temporal performance decrements for sprint distance between all first quarter intervals and 55-60 interval (Kendall's W ( $W=0.86-1.00)$, as well as the $0-5$ and all final quarter intervals for total distance $(W=0.86-1.00)$. Defenders had significant performance decreases during the 25-30 $(W=0.29-0.60)$ and $40-45$ interval $(W=0.14-0.57)$ for both total and relative distance. Forwards exhibited the lowest performance decrement, with no significant differences for peak speed, high-speed running, or sprint distance ( $W=0.00-0.53)$.

Conclusion Positional and temporal differences are evident in elite camogie match play. Mid-fielders have the greatest total and sprint distance decrements, with defenders also exhibiting total and relative distance decreases. Coaches should focus on temporal differences with respect to position, to minimise performance decrements.
\end{abstract}

Keywords GPS · Player tracking · Position · Gaelic games

\section{Introduction}

Camogie is a native Irish female field sport game. Governed by The Camogie Association, camogie is intermittent in nature, with high levels of speed, power and aerobic endurance required to compete at the elite level. Similar to the male game of hurling, it is played with a hurl (stick) and sliotar (ball). A unique skillset is required to play camogie compared to other female field sports. Teams comprise 15 players- 1 goalkeeper, 6 defenders, 2 mid-fielders and 6 forwards. Traditionally, both defending and forward units are split into two lines of three (i.e. half-forward line and

Philip Connors

philip.connors@itcarlow.ie

1 HealthCORE, Department of Science and Health, Institute of Technology Carlow, Carlow R93 V960, Ireland full-forward line). Tactical advancements in camogie have led to players in these positions frequently interchanging between the two positional lines and therefore, are identified as one within this investigation.

The Senior All-Ireland camogie championship is the pinnacle competition each year, with players who compete in this competition the most elite camogie players in Ireland. The competition structure is based on a league round-robin system, followed by knockout stages. Eleven teams compete in the competition, with a minimum of three games per team. Games are $60 \mathrm{~min}$ in duration, traditionally played in two halves of $30 \mathrm{~min}$. Due to the COVID-19 pandemic, games are now played in four 15-min quarters, with a 1-min water break after the first and third quarters. A 10-min half-time interval separates the second and third quarter.

Research on camogie and its players continues to grow in line with an increase in the sport's popularity, 
alongside players' reported time commitments and training regimes $[1,2]$. Previous research reported on players' injuries [2-4], strength and conditioning recommendations [1], and match-play demands [5, 6]. Current match-play demand research has primarily focused on half-by-half comparisons. Given the increased use and popularity of small-sided games in Gaelic sports [7], and their proposed benefits [8-10], quantifying and understanding demands for similar playing periods seem necessary, to allow appropriate comparisons, and training programming to occur. Consequently, 5-min intervals were identified as a suitable interval to achieve this outcome.

Quantifying player running load to establish training and match-play demands is commonly done via Global Positioning Systems (GPS) technology, a practically superior method of analysis compared to alternative methods [11]. GPS technology has been used across a variety of field sports including camogie, rugby union, field hockey, and soccer [5, 12-14], to track and monitor running performance. GPS metrics including total distance, high-speed running, and peak speed are commonly reported to quantify matchplay demands [15]. However, no camogie investigation has reported on running demands of shorter intervals other than halves of play. Camogie differs from other field sports, with playing numbers, playing area, and the skills required unique to the game. It is therefore not possible to utilise reports from other female field sport research, particularly given the running performance differences previously reported [5, 6].

Positional differences in running loads are evident in camogie cohorts across halves of play [5, 6]. Connors et al. [5] reported similar positional trends to elite hurling [16], with mid-fielders covering greater total distance, and forwards greater sprint distances. Anecdotally, small-sided game training interventions are conducted generically, with no positional training load differences despite positional differences during match play [5]. The absence of position-specific consideration in programme design may lead to underpreparation of some positional groups for competition.

Temporal performance decrements in field sports have been frequently reported $[14,17]$, which can be attributed to multiple factors including accumulated fatigue, tactics, and opposition quality $[17,18]$. Running reductions result in possible negative match outcomes, with higher injury rates also reported when athletes are fatigued [19]. Temporal decrements are also evident in half-by-half camogie comparisons $[5,6]$. By understanding potential periods of performance decline, coaches may make more informed tactical changes and identify appropriate time intervals to introduce substitutes.

The aim of this investigation is threefold: firstly, to investigate if positional differences occur; secondly, to identify temporal changes in performance across 5-min intervals; thirdly, to establish the match-play running demands of 5-min intervals of match play, to inform training and smallsided game prescription.

The hypothesis is that positional and temporal differences will be present, similar to previous camogie research. It is anticipated mid-fielders will cover greater distance compared to other positions, with a decline in performance noted, particularly between the first- and final-time interval, given the negative impact accumulated fatigue may have on running performance $[18,19]$.

\section{Methods}

\section{Participants}

Thirty-one $(n=31)$ senior inter-county camogie players (age: $24 \pm 4$ years; height: $167.2 \pm 6.3 \mathrm{~cm}$; body mass: $67.0 \pm 7.5 \mathrm{~kg}$ ) from two squads volunteered to participate in this investigation. Ethical approval was sought and granted by the Institutes' Research Ethics Committee. Prior to data collection, players completed health screening questionnaires and institutionally approved informed consent forms, which informed them of the study's risks, purpose, and benefits. Participants were informed they could withdraw at any time. All data collection conformed to the Declaration of Helsinki recommendations.

\section{Protocols}

To investigate the positional and temporal demands of elite inter-county camogie match play across 5-min intervals, $31(n=31)$ inter-county camogie players were recruited to participate in this investigation. Players wore $10 \mathrm{~Hz}$ Playertek (Playertek by Catapult, Australia) GPS units for all competitive games $(n=8)$ played in the Senior All-Ireland Championship. Players were split into their respective playing positions, based on match positions. 72 datasets were collected in total (defenders $(n=43)$, mid-fielders $(n=7)$, forwards $(n=22)$ ). All players completed at least 2 months training prior to data collection.

Players' height and body mass were measured in a testing session before the first game. Players stood barefoot, wearing minimal clothing, to have height and body mass assessed. Height was measured to the nearest $0.1 \mathrm{~cm}$ using a Seca Stadiometer (Seca 264, Hamburg, Germany), with body mass also measured to the nearest $0.1 \mathrm{~kg}$ using Seca Weighing Scales (Seca 704, Hamburg, Germany).

Running performance was quantified using commercially available $10-\mathrm{Hz}$ Playertek GPS units, integrated with a $400-\mathrm{Hz}$ triaxial accelerometer and a $10-\mathrm{Hz}$ triaxial magnetometer (Playertek by Catapult, Australia). Previous investigations have documented 10-Hz GPS units' validity and reliability on distance and velocities across 
linear and team sport circuits $[20,21]$. The unit (dimensions: $85 \mathrm{~mm} \times 40 \mathrm{~mm} \times 20 \mathrm{~mm}$ ) was placed in a protective pouch in the upper thoracic region between the participant's shoulder blades. GPS units were turned on 15 min prior to the warm-up to ensure satellite connection was established. Players undertook their usual pre-match warm-up which consisted of jogging, dynamic movements, and skill-related activities. GPS data were downloaded retrospectively via the Playertek software for further analysis. Games were broken into 5-min intervals using an in-built feature of the Playertek software, with three intervals per quarter. Additional time played was discarded from analysis due to the time period being less than $5 \mathrm{~min}$.

Total distance $(\mathrm{m})$, relative distance $\left(\mathrm{m} \mathrm{min}^{-1}\right)$, peak speed $\left(\mathrm{m} \mathrm{s}^{-1}\right)$, high-speed running $(\mathrm{m})$ and sprint distance (m) were analysed to quantify the players' running performance for each game. High-speed running and sprint distance thresholds were set at $4.4-5.5 \mathrm{~m} \mathrm{~s}^{-1}$ and $>5.5 \mathrm{~m} \mathrm{~s}^{-1}$, respectively, in line with previous research [22]. GPS data was downloaded from the Playertek software to a Microsoft Excel spreadsheet (Excel, Redmond, USA) for further analysis. Only players who completed a full game $(60 \mathrm{~min})$ were included in data analysis.

\section{Statistical analysis}

Data analysis was done using IBM SPSS Software 25. Data was assessed for normality using Shapiro-Wilks tests for normality. Data violated the test for normal distribution, therefore non-parametric equivalents were used.

A Kruskal-Wallis $H$ test was conducted to investigate if positional differences existed, with Bonferroni adjustments. Statistical significance was set at the accepted alpha level $p<0.05$. Temporal differences between 5-min intervals were analysed using Friedmann's test, with pairwise comparisons and Bonferroni adjustments used for post hoc analysis. To determine the magnitude of effect between positional groups, effect size $(r)$ was calculated using the standardised test statistic divided by the square root of the number of cases [23], with Kendall's W $(W)$ used for temporal estimates of effect size. Kendall's W and $r$ were interpreted using Cohen's D estimate of effect size interpretations: trivial $(<0.20)$, small $(0.20-0.49)$, moderate $(0.50-0.79)$, large (0.80-1.00) [24].

\section{Results}

Table 1 shows the descriptive statistics of positional matchplay running demands of a typical 5-min interval.

Positional analysis revealed significant differences $(p<0.05)$ between mid-fielders and other positional groups for total $(r=0.16-0.19)$ and relative distance $(r=0.15-0.17)$. Furthermore, significant differences $(p<0.05)$ between forwards and other positional groups were noted for peak speed $(r=0.14-0.18)$, high-speed running $(r=0.16-0.19)$ and sprint distance $(r=0.09-0.16)$. Trivial differences between defenders and forwards for relative distance $(r=0.02)$ was observed, with trivial differences between defenders and mid-fielders for peak speed $(r=0.04)$, high-speed running $(r=0.03)$ and sprint distance $(r=0.06)$ also.

Table 2 shows the median positional 5-min interval temporal running demands of elite inter-county camogie. Significant temporal differences $(p<0.05)$ were evident for defenders' total $(W=0.14-0.60)$ and relative distance ( $W=0.12-0.59)$, notably for the $25-30,30-35$, and $40-45$ intervals compared to intervals of the first $20 \mathrm{~min}$, as well as for the 0-5 interval compared to all third and fourth quarter intervals $(W=0.24-0.59)$. Significant differences for highspeed running between 40 and 45 and the $0-5,10-15$ and $15-20$ intervals were also observed $(W=0.09-0.46)$, with no differences across peak speed $(W=0.00-0.18)$ or sprint distance ( $W=0.00-0.28)$.

Mid-fielders had a significant decrease in performance between $0-5$ and $30-35$ intervals $(W=1.00)$, with no differences for peak speed or high-speed running. The 55-60 interval showed a significant decline in sprint performance compared to the 5-10 and 35-40 intervals $(W=1.00)$.

Table 1 Median (interquartile range) senior inter-county camogie positional running demands of a typical 5-min interval of competitive match play

\begin{tabular}{lcccc}
\hline & Overall $(n=72)$ & Defenders $(n=43)$ & Mid-fielders $(n=7)$ & Forwards $(n=22)$ \\
\hline Total distance $(\mathrm{m})$ & $450(390-520)$ & $450(380-520)^{*}$ & $490(440-540)$ & $450(380-510)^{*}$ \\
Relative distance $\left(\mathrm{m} \mathrm{min}^{-1}\right)$ & $91(77-104)$ & $89(76-103)^{*}$ & $98(87-107)$ & $91(77-103)^{*}$ \\
Peak speed $\left(\mathrm{m} \mathrm{s}^{-1}\right)$ & $6.1(5.5-6.5)$ & $6.0(5.4-6.5)^{* *}$ & $5.9(5.4-6.4)^{* *}$ & $6.2(5.7-6.7)$ \\
High-speed running distance $(\mathrm{m})$ & $40(25-59)$ & $38(22-58)^{* *}$ & $38(18-49)^{* *}$ & $44(27-62)$ \\
$\quad\left(4.4-5.5 \mathrm{~m} \mathrm{~s}^{-1}\right)$ & $14(3-29)$ & $12(1-27)^{* *}$ & $10(1-23)^{* *}$ & $19(7-34)$ \\
Sprint distance $(\mathrm{m})\left(>5.5 \mathrm{~m} \mathrm{~s}^{-1}\right)$ & & & \\
\hline
\end{tabular}

*Significantly different from mid-fielders $(p<0.05)$

**Significantly different from forwards $(p<0.05)$ 


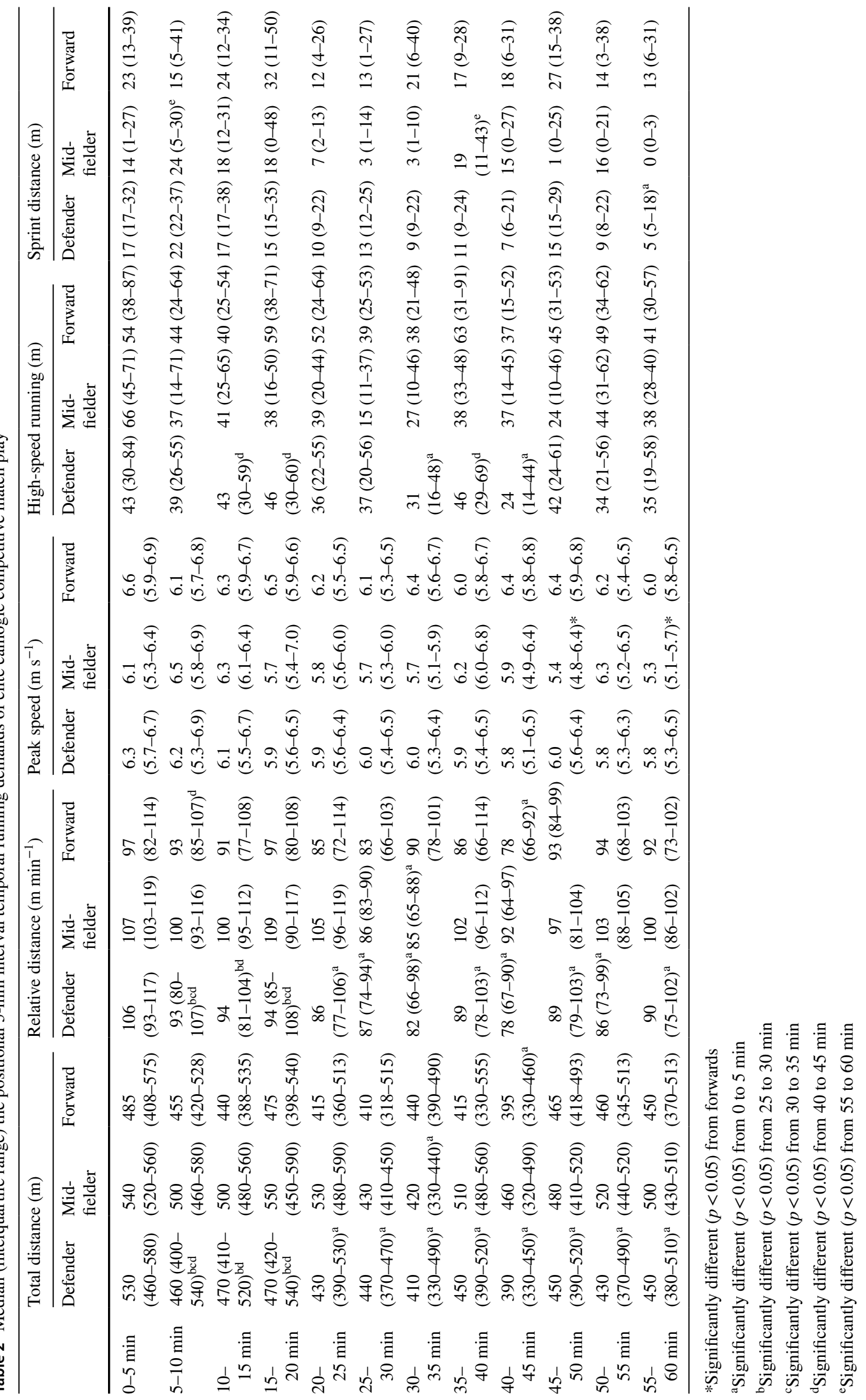


However, non-significant moderate-to-large differences were noted, notably between all intervals between $0-20$ ( $W=0.51-1.00)$ and the $25-30$ and $30-35$ intervals, and $0-5$ compared to $40-45,45-50,50-55$ and 55-60 $(W=0.51-1.00)$. There were also large differences between $35-40$, and $25-30$ and $30-35$ intervals $(W=1.00)$. Large differences in peak speed between 55-60 and 5-10, 10-15, and 35-40 were evident ( $W=1.00)$, as well as between $5-10$, and $25-30$ and $40-45$ intervals ( $W=0.86-1.00)$.

Large high-speed running performance decrements occurred at the 30-35 and 40-45 interval compared to 0-5 $(W=1.00)$, with similar decreases between $10-15$ and $25-30$ $(W=1.00)$. Further moderate differences occurred, notably between $0-5$ and $45-50,50-55$, and 55-60 $(W=0.51)$. Similar sprint distance decreases between 5-10 and 20-25 and $25-30(W=1.00)$, as well as $10-15$ and $25-30$ intervals ( $W=1.00$ ) were evident, with the 55-60 interval showing large differences to $0-5,5-10,10-15$, and 35-40 intervals $(W=0.86-1.00)$.

Only significant differences for forwards' total distance between $0-5$ and $40-45$ were noted $(W=0.49)$, with differences also between 40-45, and 5-10- and 10-15 ( $W=0.41-0.53)$. There were no other significant temporal decreases in performance across metrics for forwards, with only a moderate difference between $0-5$ and $40-45$ for high-speed running ( $W=0.53$ ). Trivial to small differences were seen for other temporal comparisons across metrics $(W=0.00-0.49)$.

\section{Discussion}

This study is the first to investigate elite camogie match-play running demands across 5-min intervals. Current camogie research has focused on half-by-half comparisons solely [5, 6]. However, with the potential application of small-sided games in training, given their use in Gaelic football cohorts [7], understanding running demands of shorter time periods seems necessary. The analysis revealed significant positional and temporal differences to exist.

Mid-fielders covered greater total and relative distance compared to other positions, similar to previous camogie research [5, 6]. Forwards cover greater high-speed running and sprint distances compared to both defenders and midfielders, as well as higher peak speeds, similar to previous camogie and field hockey investigations [5, 25]. Therefore, individualised or position-specific training is required, given obvious positional running differences. Significant temporal differences were also noted. The $0-5$ interval was most demanding for total distance, relative distance, and peak speed for defenders, with varying results for other positions. However, a general decrease in performance was noted particularly in the 5-min intervals either side of half-time and the end of the third quarter. Accumulated fatigue may be one contributing factor leading to this finding. Previous research in hurling [26], female soccer [17] and female Australian Rules football [27] have reported players to cover greater total and high-speed distance in the first quarter of match play compared to other quarters. Therefore, it should perhaps not be unexpected that performance decreases occur.

High-speed running and sprint distance account for $8.89 \%$ and $3.11 \%$ of the total distance covered. This is similar to the high-speed running percentage of the total distance covered in previous field hockey $(9.14 \%)$ and camogie studies $(9.28 \%)$, with the proportion of sprint distance covered similar to previously reported in both sports $(2.83 \%$ and $3.11 \%)[6,25]$. Relative distance is lower than that of NCAA Division I soccer (96-107 m min ${ }^{-1}$ ) and field hockey $\left(127.6 \mathrm{~m} \mathrm{~min}^{-1}\right)$ cohorts $[13,25]$, though this may be expected given a sliotar can travel up to $112 \mathrm{~km} \mathrm{~h}^{-1}$ and frequently travels greater than $50 \mathrm{~m}$, thus possibly reducing running requirements [4].

Median peak speed across intervals was $6.10 \mathrm{~m} \mathrm{~s}^{-1}$, which is lower than that reported in full game field hockey and soccer $[14,28]$. Camogie players were previously reported to achieve lower peak speeds compared to their female field sport peers [5]; therefore, this is not a novel finding. Game constraints may limit players from reaching their true peak speeds; however, further research is required to confirm this. Camogie players' mean sprint distance is $15 \pm 3 \mathrm{~m}$ [6]; therefore players may not reach their true peak speed across this distance.

Mid-fielders may require greater training workloads at lower intensities given the higher total distance covered during match play, compared with other positional groups. Forwards may benefit from greater high-speed training loads, given the greater distance covered at higher speeds during match play compared to other positions. Although defenders and forwards covered similar total and relative distance, defenders completed less high-speed running and sprinting in comparison. This may be attributed to the skills and positioning associated with defending, alongside the need to anticipate their opponents' movement [15, 25]. Therefore, although total positional running load is similar, there is perhaps a requirement to focus on anticipatory and defenderspecific work also.

Temporal reductions in performance are cited as a possible injury risk [19], with higher injury rates reported in a game's final third compared to other time periods [29]. Therefore, reducing or better understanding this performance decrement is necessary. Total and relative distance decrements of 19-26\% were apparent across positions from the highest-to-lowest 5-min intervals. Larger sprint decrements were also evident (59-100\%), with mid-fielders having the largest high-speed running and sprint distance decrements. These decrements are higher than previously reported 
in elite camogie (14.84\% and 29.90\%) [5]. Although external factors such as opposition or result may impact temporal decrements, this is more likely applicable to single games and may be negated across a championship campaign. Given the negative impact of decrements on performance, alongside the increased injury risk, attempts to reduce these decrements should be prioritised.

The end of the third quarter (40-45 min), alongside both 5-min intervals either side of half-time exhibits the largest total and relative distance decrement in defenders' performance. The reasons for this are unclear, given an increase in total distance was evident in later time intervals. Similar increases during the final quarter of match play have been previously reported in field hockey reports [25]. It is plausible an increase in running performance in the final quarter may be due to substitute introduction. Substitutions have previously been reported to have greater relative work rate profiles in both Gaelic football [30] and NCAA Division I soccer [13] games compared to full game players. Therefore, it may be that full game players' attempts to match substitutes' running performance leads to an increase in running performance in the final quarter.

Mid-fielders had the largest decrement in performance across positions, with this similar to previous camogie reports [5,6]. This decrement was most notable for highspeed running and sprint distance. This is an area coaches may look to address through appropriate training methods. Mid-fielders had a large decrease in total and relative distance during the last $15 \mathrm{~min}$ of match play compared to the first $5 \mathrm{~min}$, with decreases also noted during the interval either side of half-time and the final interval of the third quarter. Furthermore, mid-fielders had large decreases in peak speed across intervals, unlike defenders.

Forwards were the most consistent positional group across time intervals. However, performance decreases were again observed at the end of the third quarter for total and relative distance. No significant differences were noted for peak speed, high-speed running, or sprint distance. A greater distance covered at higher speeds and lower total distance compared to mid-fielders may allow forwards to recover fully between bouts, thus reducing performance decrements. Also, the frequent interchanging of positional lines may result in some forwards spending greater time in the full-forward line, a position which covers a similar number of sprints, but lower total distance compared to the half-forward line [6].

Furthermore, performance decreases prior to half-time may be due to accumulated fatigue over the $30 \mathrm{~min}$, with half-time passive nature perhaps hindering performance during the $5 \mathrm{~min}$ after the interval. Half-time has a reported negative impact on performance, with a re-warm-up attenuating the reduction in performance in male soccer players [31]. Future research may investigate the effect of a re-warm-up on performance in camogie. The intervals with reduced performance may provide an area for coaches to target, given a possible reduced workload for full game players during these periods, as well as the impact of substitutions on running performance.

Running performance may be impacted by technical performance indicators [32], therefore tracking the running performance of one squad of players may result in biased results due to a team's tactics, playing style and skill levels. However, in tracking different camogie squads, this investigation attempted to negate these limitations. These results provide normative positional running demand ranges which may be more applicable to programme small-sided games in training. The benefits of these games have been widely documented in hurling cohorts $[8,9]$; however, further research may look to examine their use in camogie. Further research on club (sub-elite) match play may also be examined, as well as assessing the impact of technical performance indicators on running performance through video analysis. Positional differences are evident during elite camogie match play; therefore, attempts to prepare players appropriately according to their position is necessary.

\section{Conclusion}

This study is the first to provide a more in-depth running demand analysis of elite camogie players. Positional variation is apparent, with mid-fielders covering greater total and relative distance compared to other positions, and forwards covering greater distances at high-speed and sprint distances, as well as achieving higher peak speeds. Temporal performance decrements were observed, with midfielders exhibiting the largest decrements in performance. Both defenders and mid-fielders had significant performance decreases during the $25-30$ and 40-45 intervals, with midfielders' total distance decreasing during the final three intervals. Forwards had smaller decrements in performance compared to other positions. Coaches should consider positional running differences, along with temporal performance decrements when programming training and planning substitutions during match play. Small-sided games are a popular training intervention with short time periods of play commonly utilised. Players should reach similar or greater distances to that reported during a 5-min interval of match play to ensure adequate preparation for match-play demands.

Large temporal decreases are evident, particularly for defenders and mid-fielders. Coaches may attempt to reduce these decrements through appropriate training methods alongside tactically utilising substitutions. A re-warm-up protocol prior to the start of the second half may also aid in reducing the performance decrement noted within that time period.

Acknowledgements The authors wish to acknowledge the respective camogie squads and players who participated in this investigation. 
Funding This research was funded by the Institute of Technology Carlow President's Research Fellowship Scholarship Award.

Data availability The data associated with this manuscript is available from the corresponding author (P. Connors) upon reasonable request.

\section{Declarations}

Conflict of interest The authors report no potential conflict of interest.

Ethical approval The Institute of Technology Carlow's Research Ethics Committee approved all procedures (Ethical Approval No: 253), and the study was conducted according to the Declaration of Helsinki (1975) for human subject studies.

Informed consent Informed consent was obtained from all individual participants included in the study.

\section{References}

1. Duggan J, Moody J, Byrne P, Ryan L (2020) Strength and conditioning recommendations for female GAA athletes: the camogie player. Strength Cond J 42(4): 105-124

2. O'Connor S, Lacey P (2020) Can we improve coaches' injury prevention views and implementation practices in the community female Gaelic sport of camogie? BMJ Open Sport Exerc Med 6(1): $\mathrm{e} 000732$

3. Buckley C, Blake C (2018) The incidence of injury in elite camogie, an in-season prospective study. BMJ Open Sport Exerc Med 4(1):e000315

4. O'Connor S, Leahy R, Whyte E, Donovan P, Fortington L (2019) Understanding injuries in the Gaelic sport of camogie: the first national survey of self-reported worst injuries. Int J Athl Ther Train 24:243-248

5. Connors P, Earls D, Browne D, Fitzpatrick P, Rankin P (2020) The positional demands of inter-county camogie. Sport Perf Sci 104(1):1-2

6. Young D, O'Grady M, Coratella G (2020) The match-play running performance of elite camogie players across halves of play. Sport Sci Health 17(1):191-199

7. Kinnerk P, Harvey S, Kearney P, MacDonncha C, Lyons M (2019) An investigation of the self-reported practice activities and session sequencing of inter-county Gaelic football coaches. Int Sport Coach 6(2):1-9

8. Malone S, Collins K (2017) Effect of game design, goal type and player numbers on the physiological and physical demands of hurling-specific small-sided games. J Strength Cond Res 31(6):1493-1499

9. Malone S, Hughes B, Collins K (2019) The influence of exercise-torest ratios on physical and physiological performance during hurling-specific small-sided games. J Strength Cond Res 33(1):180-187

10. Mangan S, Collins K, Burns C, O'Neill C (2019) An investigation into the physical and technical demands of small-sided games using varying pitch dimensions in Gaelic football. Int J Perf Anal Sport 19(6):971-984

11. Johnston R, Watsford M, Pine M et al (2012) The validity and reliability of $5-\mathrm{Hz}$ global positioning system units to measure team sport movement demands. J Strength Cond Res 26(3):758-765

12. Bradley EJ, Board L, Hogg B, Archer D (2020) Quantification of movement characteristics in women's English premier elite domestic rugby union. J Hum Kinet 72(1):185-194
13. Vescovi J, Favero T (2014) Motion characteristics of women's college soccer matches: Female Athletes in Motion (FAiM) study. Int J Sports Physiol Perform 9(3):405-414

14. Vescovi J, Frayne D (2015) Motion characteristics of Division I college field hockey: Female Athletes in Motion (FAiM) study. Int J Sports Physiol Perform 10(4):476-481

15. Hodun M, Clarke R, De Ste CM, Hughes J (2016) Global positioning system analysis of running performance in female field sports: a review of the literature. Strength Cond J 38(2):49-56

16. Young D, Beato M, Mourot L, Coratella G (2019) Match-play temporal and position-specific physical and physiological demands of senior hurlers. J Strength Cond Res 34(6):1759-1768

17. Hewitt A, Norton K, Lyons K (2014) Movement profiles of elite women soccer players during international matches and the effect of opposition's team ranking. J Sports Sci 32(20):1874-1880

18. White A, MacFarlane N (2015) Contextual effects on activity profiles of domestic field hockey during competition and training. Hum Mov Sci 40:422-431

19. Small K, McNaughton L, Greig M, Lovell R (2010) The effects of multi-directional soccer-specific fatigue on markers of hamstring injury risk. J Sci Med Sport 13(1):120-125

20. Beato M, Coratella G, Stiff A, Iacono A (2018) The validity and between-unit variability of GNNS units (STATSports Apex 10 and $18 \mathrm{~Hz}$ ) for measuring distance and peak speed in team sports. Front Physiol 9:1288

21. Coutts AJ, Duffield R (2010) Validity and reliability of GPS devices for measuring movement demands of team sports. J Sci Med Sport 13(1):133-135

22. Bradley P, Vescovi J (2014) Velocity thresholds for women's soccer matches: sex specificity dictates high-speed running and sprinting thresholds- Female Athletes in Motion (FAiM). Int J Sports Physiol Perform 10(1):112-116

23. Pallant J (2011) SPSS survival manual: a step by step guide to data analysis using the SPSS program, 4th edn. Allen \& Unwin, Berkshire

24. Cohen J (1988) Statistical power analysis for the behavioral sciences. Routledge Academic, New York

25. McGuinness A, Malone S, Hughes B, Collins K, Passmore D (2019) Physical activity and physiological profiles of elite international female field hockey players across the quarters of competitive matchplay. J Strength Cond Res 33(9):2513-2522

26. Young D, Kilty J, Hennessy L, Coratella G (2020) The running performance decrement in elite hurling. Appl Sci 10(22):8191

27. Clarke A, Ryan S, Couvalias G, Dascombe B, Coutts A, Kempton $\mathrm{T}$ (2018) Physical demands and technical performance in Australian Football League Women's (AFLW) competition match-play. J Sci Med Sport 21(7):748-752

28. Vescovi J (2012) Sprint profile of professional female soccer players during competitive matches: Female Athletes in Motion (FAiM) study. J Sports Sci 30(12):1259-1265

29. Kofotolis N, Kellis E, Vlacjopoulos S (2007) Ankle sprain injuries and risk factors in amateur soccer players during a 2-year period. Am J Sports Med 35(3):458-466

30. Boyle E, Warne J, Nevill A, Collins K (2020) The work-rate of substitutes in elite Gaelic football match-play. Sports Perf Sci 89(1):1-3

31. Edholm P, Krustrup P, Randers M (2014) Half-time re-warm up increase performance capacity in male elite soccer players. Scand J Med Sci Sports 25(1):40-49

32. Mangan S, Ryan M, Devenney S et al (2017) The relationship between technical performance indicators and running performance in elite Gaelic football. Int J Perform Anal Sport 17(5):706-720

Publisher's Note Springer Nature remains neutral with regard to jurisdictional claims in published maps and institutional affiliations. 